\title{
Unusual subpulse modulation in PSR B0320+39
}

\author{
R. T. Edwards ${ }^{1}$, B. W. Stappers ${ }^{1,2}$, and A. G. J. van Leeuwen ${ }^{3}$ \\ 1 Astronomical Institute “Anton Pannekoek”, University of Amsterdam, Kruislaan 403, 1098 SJ Amsterdam, The Netherlands \\ 2 Stichting ASTRON, Postbus 2, 7990 AA Dwingeloo, The Netherlands \\ 3 Astronomical Institute, Utrecht University, Postbus 80000, 3508 TA Utrecht, The Netherlands
}

Received 28 June 2002 / Accepted 20 February 2003

\begin{abstract}
We report on an analysis of the drifting subpulses of PSR B0320+39 that indicates a sudden step of $~ 180$ degrees in subpulse phase near the centre of the pulse profile. The phase step, in combination with the attenuation of the periodic subpulse modulation at pulse longitudes near the step, suggests that the patterns arise from the addition of two superposed components of nearly opposite drift phase and differing longitudinal dependence. We argue that since there cannot be physical overlap of spark patterns on the polar cap, the drift components must be associated with a kind of "multiple imaging" of a single polar cap "carousel" spark pattern. One possibility is that the two components correspond to refracted rays originating from opposite sides of the polar cap. A second option associates the components with emission from two altitudes in the magnetosphere.
\end{abstract}

Key words. magnetic fields - plasmas - pulsars: individual: PSR B0320+39 - waves

\section{Introduction}

For a number of pulsars it is known that each pulse is composed of a number of subpulses, and that for each successive pulse the subpulses appear to "drift" by a given amount across the profile (Drake \& Craft 1968). The rate at which subpulses drift across the profile is not constant but rather depends on their pulse longitude. This can be understood in the context of the widely adopted "carousel" model of drifting subpulses (Ruderman 1972), which postulates the existence of a ring of equally spaced "sparks" above the polar cap that give rise to "tubes" of plasma streaming upward from the surface, ducted along magnetic field lines. At a certain altitude these particles emit microwave radiation that is beamed along tangents to the local field lines, giving rise to a beam pattern that consists of a ring of "subbeams" (bright spots), reflecting the polar cap spark configuration. As the pulsar rotates the distant observer samples emission along a certain path in the beam pattern. Under the right viewing geometry, the sight-line makes a tangential pass along the ring of subbeams, giving rise to the reception of a sequence of one to a few subpulses every time the star rotates. Due to the non-linear mapping between (spin) longitude and magnetic azimuth, the subpulses should appear more closely spaced around the point at which the sight-line makes its closest approach to the magnetic pole. As the carousel slowly rotates about the magnetic pole, the longitude at which the emission from a given spark is seen exhibits a monotonic drift with time, which by extension of the above argument is greater in magnitude further from the magnetic pole.

Send offprint requests to: $\mathrm{R}$. T. Edwards, e-mail: redwards@astro.uva.nl
By stacking the intensity time series modulo the pulse period to produce a two-dimensional array in pulse number and pulse longitude, and taking Fourier transforms along constantlongitude columns (forming a longitude-resolved fluctuation spectrum; LRFS), Backer (1970a,b) was able to measure not only the characteristic periodicity in pulse number $\left(P_{3}\right.$, the time taken for one subpulse to drift to the former position of its neighbour), but also its amplitude and phase as a function of pulse longitude. Many subsequent studies employed this method but ignored the phase information, however those that examined the longitude-phase relation (Backer 1970a; Wright 1981; Davies et al. 1984; Biggs et al. 1987; Ashworth 1988) found behaviour qualitatively consistent with that expected under the carousel model.

The longitude-phase relationship expected under the carousel model depends on the number of sparks present, the misalignment angle between the spin and magnetic axes, and their orientation with respect to the line of sight. Hence studies of the relationship have the potential to provide valuable information about the pulsar, particularly in comparison to the observed longitudinal dependence of the polarisation position angle which, under the magnetic pole model of Radhakrishnan \& Cooke (1969), depends on the same geometric parameters.

Motivated by these goals, we embarked on a program of analysis of several pulsars with drifting subpulses using the Westerbork Synthesis Radio Telescope (WSRT). One of the target sources was PSR B0320+39. This pulsar was discovered independently by Damashek et al. (1978) and Izvekova et al. (1982) in surveys of the northern sky at 400 and $102.5 \mathrm{MHz}$ respectively. Further observations revealed the emission of very regular drifting subpulses 


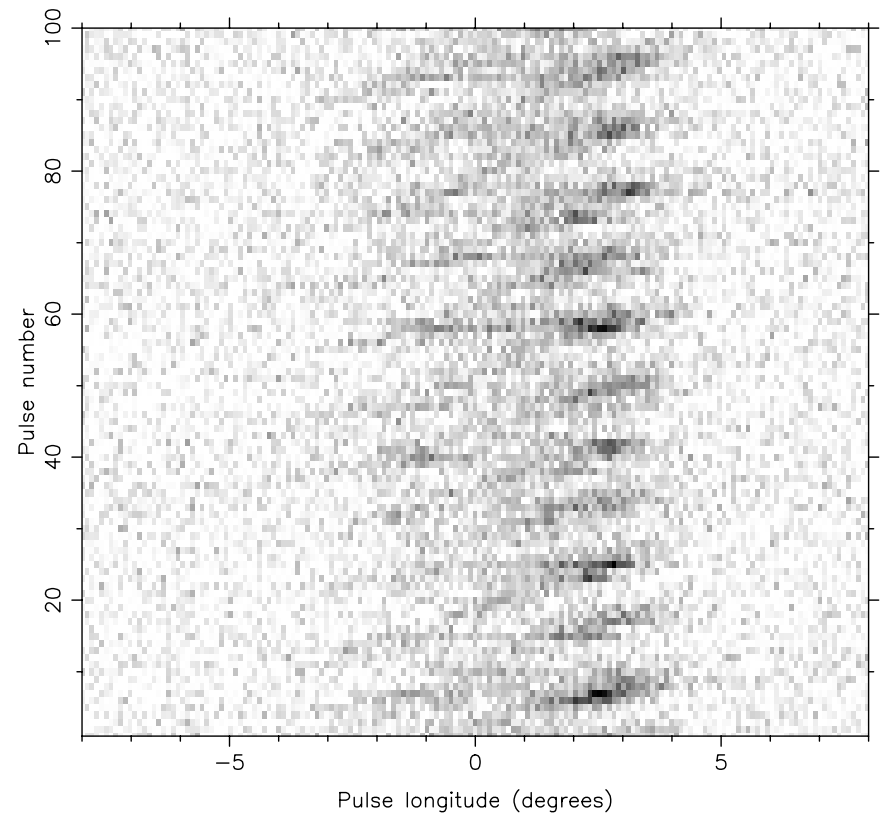

Fig. 1. First 100 pulses of $328 \mathrm{MHz}$ timeseries for PSR B0320+39, binned in pulse longitude and pulse number.

(Izvekova et al. 1982) which occur in two distinct pulse longitude intervals, with a non-drifting component present in the average profile (Izvekova et al. 1993; Kouwenhoven 2000). In this paper we report a most unexpected result from our study of the longitude-phase relationship of the drifting subpulses of PSR B0320+39.

\section{Observations and analysis}

We observed PSR B0320+39 on 2000 November 3 with the Westerbork Synthesis Radio Telescope (WSRT). Signals from fourteen telescopes were added (after appropriate delays) in each sense of linear polarisation in a $10 \mathrm{MHz}$ band centred at $328 \mathrm{MHz}$. These were processed using the Dutch Pulsar Machine (PuMa), operating as a digital filterbank with 64 channels and $819.2 \mu$ s sampling in total intensity (Stokes I). For details of the system see Voûte et al. (2002).

In offline analysis we de-dispersed the data and binned the resultant time series into an array in pulse longitude and pulse number using the ephemeris of Arzoumanian et al. (1994). A total of 3400 pulses in 3701 longitude bins was recorded, with a region of 512 bins including the on-pulse used in further analysis. The samples were normalised to produce an average profile with a zero baseline and a peak value of unity. The first 100 pulses are shown in a greyscale form in Fig. 1.

The techniques employed in the further analysis are based on those presented in detail by Edwards \& Stappers (2002). We computed the two-dimensional Discrete Fourier Transform (DFT) of the first 2048 pulses of the pulse longitude - pulse number array to yield what we call the two-dimensional fluctuation spectrum (2DFS). This is mathematically equivalent to the harmonic resolved fluctuation spectrum (HRFS;

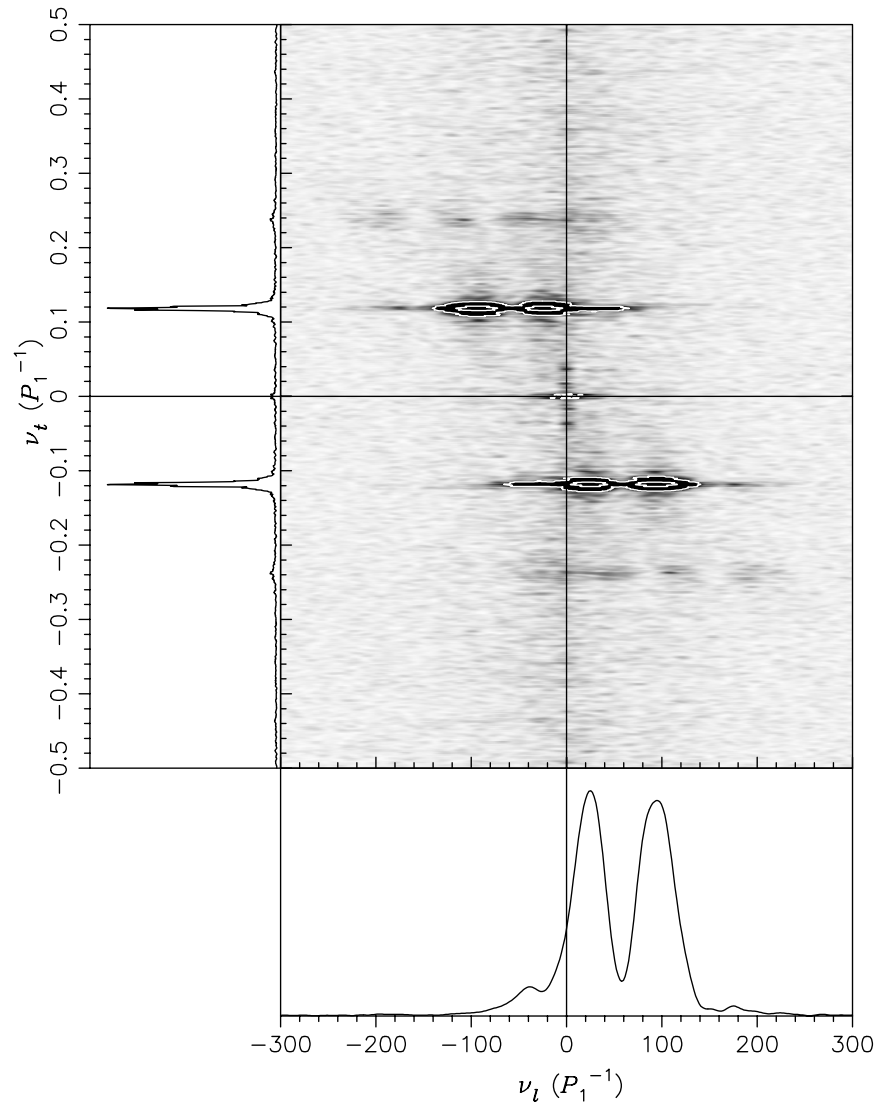

Fig. 2. Main panel: two-dimensional fluctuation power spectrum of PSR B0320+39. The greyscale saturates at 0.05 times the peak power, while the contours show the same spectrum at levels 0.05 and 0.5 times the peak. Left panel: "collapsed" spectrum formed by averaging rows of the $2 \mathrm{D}$ spectrum. Bottom panel: power as a function of $v_{\mathrm{l}}$ for $v_{\mathrm{t}}=-0.118$.

Deshpande \& Rankin 1999; Edwards \& Stappers 2002) ${ }^{1}$, and the form of the power spectrum found here for PSR B0320+39 matches that found by Kouwenhoven (2000) for the HRFS. For the purposes of clear display we also computed a spectrum using data from which we subtracted the average profile from each pulse, and padded with zeros in the longitude axis, in order to improve the resolution. The resolution in the time-associated axis was unnecessarily high (2048 elements), so to improve the signal-to-noise ratio we smoothed the power spectrum by convolution with a $7 \times 1$ bin $\left(v_{\mathrm{t}} \times v_{1}\right)$ boxcar before plotting the relevant portion of the spectrum ${ }^{2}$ in Fig. 2. The spectrum is dominated by a pair of components associated with the drift modulation; two are present because the spectrum derives from the Fourier transform of real-valued data (see Edwards \& Stappers 2002). Also present with high significance are a pair of responses

\footnotetext{
1 Without the use of "twiddle factors" (Cooley \& Tukey 1965), the 2DFS will differ from the HRFS. However, it is easily shown that this merely amounts to the provision of slightly different sampling lattices over the same continuous function, due to the assignment of integer pulse numbers (2DFS) versus integer harmonic numbers (HRFS).

2 The sampling interval used allows longitude-associated frequencies up to 1850 cycles/period, however all significant power is present in the portion of the spectrum shown.
} 
corresponding to the second harmonic of the subpulse response. The features near $v_{\mathrm{t}}=0.02$ and 0.04 cycles/period are significant, however they also appear in an analysis of off-pulse data, indicating that they are caused by periodic interference.

After performing the 2D DFT, the complex spectrum was shifted in both axes by an amount which placed the primary response to the drifting subpulses at zero frequency. We then masked other components present in the spectrum (namely the complex conjugate "mirror" of the primary response, the pair of second harmonics and the DC component) by multiplication of the coefficients with a transfer function:

$f\left(v_{\mathrm{t}}\right)=\left\{\begin{array}{ll}0 & \left|v_{\mathrm{t}}-v_{\mathrm{tc}}\right| / w<0.5 \\ 2\left|v_{\mathrm{t}}-v_{\mathrm{tc}}\right| / w-1 & 0.5 \leq\left|v_{\mathrm{t}}-v_{\mathrm{tc}}\right| / w \leq 1 \\ 1 & \left|v_{\mathrm{t}}-v_{\mathrm{tc}}\right| / w>1\end{array}\right.$,

where $v_{\mathrm{t}}$ is the time-associated axis, $v_{\mathrm{tc}}$ is the centre frequency of the component to mask, and $w$ is the frequency extent of the region of zero transmission. The results that follow derive from filters with $w=0.05$ cycles/period, although as expected they were found to be insensitive to $w$ unless it becomes too small to mask adequately or so large that it masks some of the desired signal. We then computed the inverse DFT of the result.

We represent the subpulse signal as the real part of the product of a pure two-dimensional sinusoid and a two-dimensional complex modulation envelope. By the convolution theorem, this envelope is the result of the inverse transform performed above. It describes the deviations from a pure periodicity of constant amplitude that arise due to amplitude modulation at the pulse period, scintillation or nulling, non-uniform spacing of subpulses in pulse longitude (e.g. due to sight-line curvature) and slow variations in the drift rate. Using the iterative scheme of Edwards \& Stappers (2002) we decomposed the two-dimensional envelope into the product of two modulation envelopes that vary in pulse longitude and pulse number respectively. Such a decomposition is able to model the behaviour of a rotating carousel, where the geometry defines the longitude-phase dependence and the carousel rotation determines the time-phase dependence, and its basic assumptions - that the longitudinal spacing of the subpulses is time invariant, and that the time spacing of the subpulses is longitude independent - are in agreement with previous studies; see Edwards \& Stappers (2002). The validity of this approach in the case of PSR B0320+39 was confirmed by computation of the 2DFS of the difference between the observed signal and that predicted using the real part of product of the two one-dimensional envelopes. No significant power remained in the portion of the spectrum previously occupied by the primary drifting subpulse response.

The spectral shifting performed earlier has the effect of removing a constant phase slope in each of the envelopes. Since the slope varies over the envelope, the choice of value to use in the shifting is somewhat arbitrary and was refined after viewing the resultant envelopes. The periodicity with pulse number was well-defined and a value of $\hat{P}_{3}=-P_{1} / 0.118$ was used. The response to the drifting component in the other axis is very broad, and in fact extends well into negative frequencies (Fig. 2). This complicates the choice of value for $1 / \hat{P}_{2}$ to be used in shifting the spectrum in the longitude axis. We chose a value of

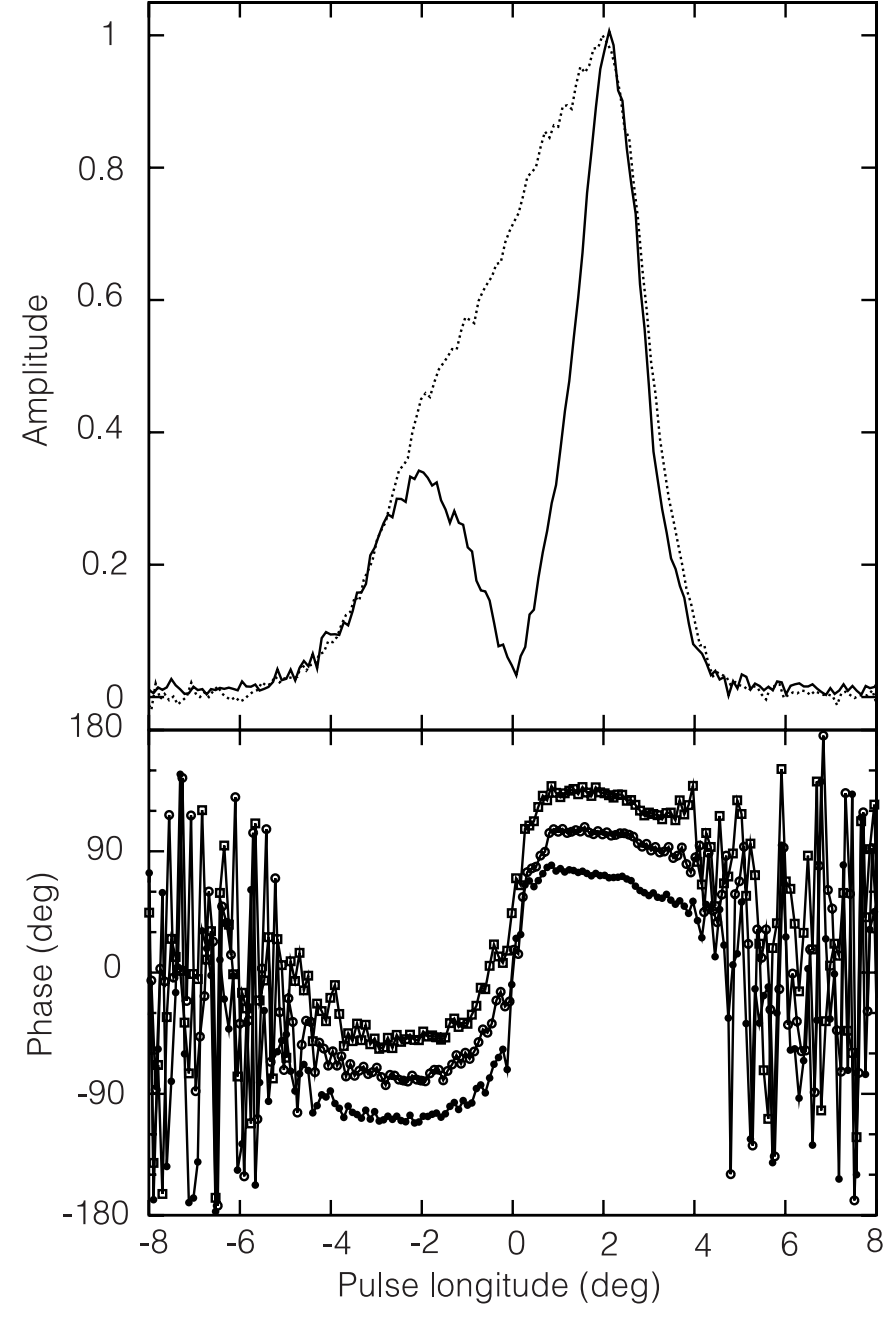

Fig. 3. Inferred modulation envelope (solid line and filled circles) and average pulse profile (dotted line). Also shown is the phase envelope computed by coherent addition of a set of 69 rows $\left(0.108<v_{\mathrm{t}}<\right.$ 0.128 ) of the 3400-pt LRFS over which significant power was seen (unfilled circles), and the phase envelope computed by coherent addition of the peak row from each of twenty-six 128-pt LRF spectra resulting from time-segmentation of the sequence (with this transform length the majority of modulation power falls in a single row). The nominal phase slope of $1 / \hat{P}_{2}=60^{\circ} \%^{\circ}$ has been subtracted from the phase envelopes before plotting.

$\hat{P}_{2}=P_{1} / 60$ to give the resultant envelope a relatively small phase slope over most of the pulse $\mathrm{s}^{3}$.

The inferred longitude-dependent envelope (Fig. 3) shows that nearly linear subpulse modulation occurs in two distinct longitude intervals, with a striking phase offset of $\sim 180^{\circ}$ between them. In terms of the longitude-time diagram, the effect of the offset is to cause the drift band peaks in one component to extrapolate to troughs in the other and vice versa. To check this result we computed the (complex) LRFS and examined the amplitudes and phases in frequency bins near $\hat{P}_{3}=P_{1} / 0.118$. Since the modulation is not a pure

\footnotetext{
${ }^{3}$ Since this value does not lie on the natural grid of the DFT, the shift was actually performed in the time-longitude domain by multiplication with a complex exponential.
} 


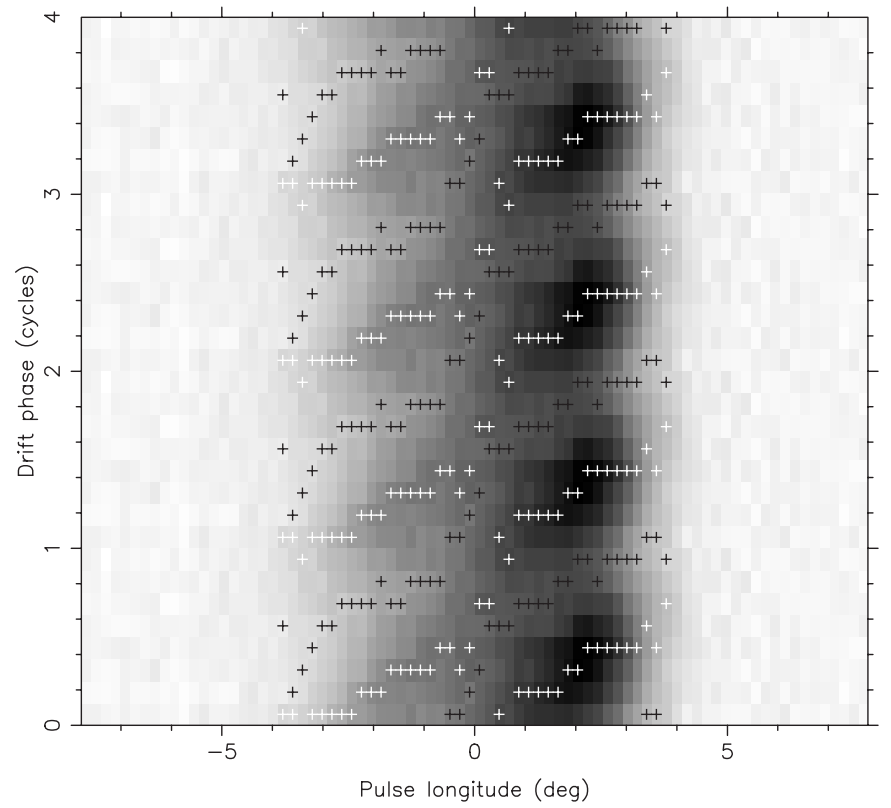

Fig. 4. Data folded at $P_{3}$, as a function of pulse longitude and drift phase. Maxima and minima in each longitude bin are indicated with black and white crosses respectively. The data are plotted four times to guide the eye.

periodicity, the power is spread over several bins in the spectrum. Although of a much lower signal-to-noise ratio, the shape of the phase envelope in each bin was consistent with that inferred with our preferred technique, which makes optimal use of all modulation power. Similarly, division of the observation into segments in time (which results in wider frequency bins and potentially also in reduced frequency drift in each segment) and examination of the phase relation in the peak bin of each segment also gave consistent results (regardless of the segment size). In order to improve the signal-to-noise ratio of these other methods, we also used an iterative algorithm based on multiplication with the complex conjugate of a template envelope to determine the correct phases with which to add envelopes from different frequency bins or time segments (producing in effect coarse-grained versions of the algorithm of Edwards \& Stappers 2002). The resultant summed envelopes were again consistent with the result of our preferred algorithm (Fig. 3). As a final check, we folded the longitude-time data about the pulse number axis (modulo $1 / 0.118$ pulses) to produce an average profile as a function of drift phase and pulse longitude (Fig. 4). As with the LRFS methods, much of the modulation was lost due to the unstable drift motion, however some residual modulation remained and is sufficient to confirm the unusual phase behaviour.

Knowing that there is a $\sim 180^{\circ}$ offset between the two halves of the subpulse phase envelope, we may explain two otherwise curious facts about PSR B0320+39. The first concerns the values of $P_{2}$ previously reported, which are around $P_{1} / 120$ (Izvekova et al. 1993 report $3.1^{\circ}$ of longitude, i.e. $P_{2} \simeq P_{1} / 116$ at $406 \mathrm{MHz}$; Kouwenhoven 2000 reports $24.5 \mathrm{~ms}$, i.e. $P_{2} \simeq$ $P_{1} / 123$, at $328 \mathrm{MHz}$ ). These strongly differ from our nominal $\hat{P}_{2}=P_{1} / 60$ because they are based on measurements of actual subpulse separations, which will nearly always be taken between subpulses on opposite sides of the phase shift. Since the subpulse in the trailing component leads (or lags) the position it would take in a continuous linear phase envelope by $\sim 180^{\circ}$ of subpulse phase, the derived spacing differs from that expected from the typical phase slope by a factor of $1 / 2$ (and probably sometimes $3 / 2$ ). The second curiosity explained by the phase offset is the local minimum in the subpulse HRFS response at around 60 cycles/period (Fig. 2). The complex longitude-dependent modulation envelope (once the constant phase slope is subtracted) is quite close to an odd realvalued function, which causes its Fourier transform to have local minimum in its power at DC. This is the cause of the local minimum around 60 cycles/period, the typical longitudinal drift frequency. Without the phase offset the envelope would be close to a real-valued even function and its Fourier transform would not have a strong minimum at its centre.

Furthermore, we note that the equivalence of the 2DFS and HRFS provides an explanation for the apparent "secondary" component at 0.118 cycles/period, suggested by Kouwenhoven (2000) to be due to the ambiguity between which drift band joins to which between the two components. In fact, the subpulse response is very broad in the longitude-associated frequency axis, and extends significantly into negative frequencies. The symmetry of the two dimensional Fourier transform of real functions is such that the power at any frequency pair $v_{1}, v_{\mathrm{t}}$ is equal to that at $-v_{1},-v_{\mathrm{t}}$ (where $v_{1}$ and $v_{\mathrm{t}}$ are the frequencies in the longitude-associated and time-associated axes respectively). If only the positive frequencies in $v_{1}$ are plotted, as is the case with the HRFS, no information is lost but individual components that span the $v_{1}=0$ axis will appear as two spatially separated components at $v_{\mathrm{t}}$ values of opposite sign. When the full spectrum is plotted as in Fig. 2 the proper interpretation becomes apparent.

\section{Discussion and interpretation}

\subsection{Polar cap models}

The phenomenon of drifting subpulses has been used repeatedly in investigations of pulsar emission as a potentially strong clue about the geometry of the system. The fact that those pulsars that show nearly linear drifting subpulses tend to have a double, "unresolved double" or "single" average pulse profile morphologies, with many showing a transition from resolved to unresolved double as the observing frequency is increased, is usually seen as strong support for some form of "hollow-cone" polar cap model (Radhakrishnan \& Cooke 1969; Ruderman \& Sutherland 1975; Backer 1976; Rankin 1983; Rankin 1986; Lyne \& Manchester 1988). PSR B0320+39 shares these characteristics of regular drifting subpulses and a transition from a resolved double to an unresolved double average profile morphology between $102 \mathrm{MHz}$ (Izvekova et al. 1982) ${ }^{4}$ and $328 \mathrm{MHz}$ (this work; also Damashek et al. 1978; Izvekova et al. 1993).

\footnotetext{
4 Due to an improved system response the profile of Kuz'min \& Losovskii (1999) shows much stronger component separation than that of Izvekova et al. (1982); see Fig. 6.
} 
The local steepening of the phase envelope of PSR $\mathrm{B} 0320+39$ in the transition region is inconsistent with the carousel model in its most basic form. For a single ring of "sparks", as long as the sparks are elongated in either magnetic azimuth or opening angle (or neither, but not both), the subpulse modulation seen in a ring of constant opening angle has the same phase as that in any other ring, if measured from the same fiducial azimuth. Therefore, the observed longitude-dependent subpulse phase envelope is directly tied to to the magnetic azimuth sampling of the sight-line (via multiplication by the number of sparks, $N$ ) (Edwards \& Stappers 2002). The only way to produce a sudden swing in magnetic azimuth (and hence subpulse phase) is to pass close to the magnetic pole. Such a configuration is inconsistent with the usual interpretation of unresolved double profile morphologies, and with the gradual linear polarisation position angle swing observed in this pulsar (Suleimanova \& Pugachev 2002). To prove this assertion we attempted to fit the observed subpulse phase swing with the geometric formula of Edwards $\&$ Stappers (2002), using a $\chi^{2}$ minimisation algorithm. From the figure provided by Suleimanova \& Pugachev (2002) we conservatively estimate the range of polarization position angle gradients consistent with the data as $\mathrm{d} \chi / \mathrm{d} \phi=12 \pm 3^{\circ} \%^{\circ}$ (where $\chi$ and $\phi$ are the position angle and pulse longitude respectively). The parameters of the fit were required to be consistent with this gradient via the geometric model of Radhakrishnan \& Cooke (1969). The result (Fig. 5) clearly fails to describe the observed behaviour.

One way to produce a jump in subpulse phase is to adopt a nested ring polar cap spark configuration (Gil \& Sendyk 2000) and to assign the two halves of the profile to rings of different opening angles and numbers of sparks. However, this would result in a measurably different $P_{3}$ unless the circulation rates of the two carousels were different by a fortuitous ratio (i.e. the inverse of the ratio of the numbers of sparks in the rings). In addition, the transition from resolved to unresolved double profile with increasing frequency could not be explained by standard radius-to-frequency mapping (RFM; Cordes 1978). Some other mechanism such as the finite beam-width of the elementary emission mechanism $\left(2 \gamma^{-1}\right)$, the finite band-width of emission at a given radius (with attendant RFM in the effective range of radii visible), or refractive broadening would be required to produce the profile evolution.

\subsection{Double imaging of magnetospheric origin}

\subsubsection{Basic considerations}

Given the failure of the standard model to account for the observed subpulse drift behaviour of PSR B0320+39, we develop in this section a pair of explanations based on a simple and plausible extension of the model. Namely, we argue that the most important features of the longitude-dependent subpulse modulation envelope - a local reduction of amplitude accompanied by a rapid swing in the phase angle - are the result of destructive interference between two superposed drifting subpulse signals of nearly opposite phase. The observed behaviour and this explanation of it can be understood by analogy with

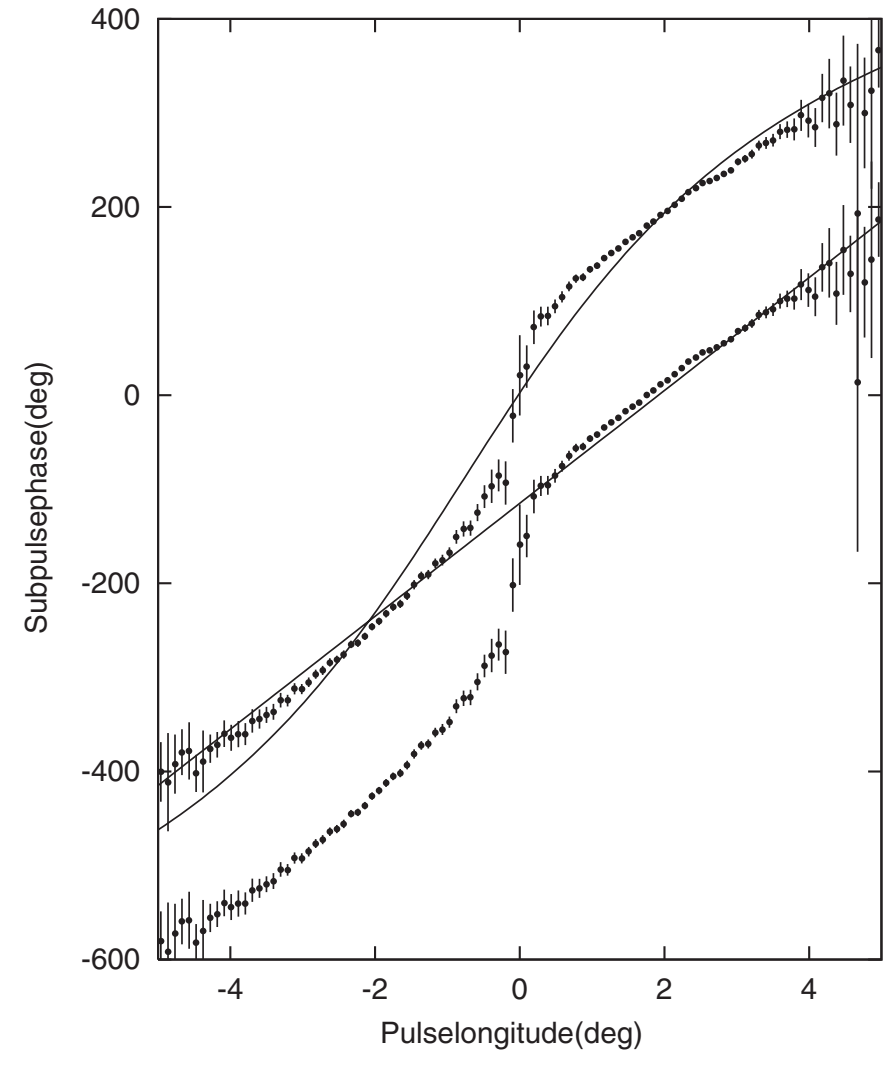

Fig. 5. Inferred subpulse phase envelope, including the $60^{\circ} /{ }^{\circ}$ phase slope previously removed (indicated by the straight line). The data are plotted twice with an offset of $180^{\circ}$ to facilate evaluation of the central feature as the transition between two offset regions of linear drift. Two-sigma error bars are included. The curved line represents the best fit to the data under the standard carousel model, given the polarimetric constraint (see text).

the phenomenon of orthogonal polarisation mode transitions, where the pulsar signal is seen to consist of the incoherent sum of two signals with nearly orthogonal polarisation states (e.g. Manchester et al. 1975; Stinebring et al. 1984). In both cases the total intensity of the two signals simply add in the average profile, while the near anti-parallel nature of the vectors representing the complex subpulse modulation signal (in the Argand plane) or the polarised part of the signal (in the Poincaré sphere) explains the reduction in magnitude (subpulse amplitude, polarised intensity) and the swing in orientation (subpulse phase, polarisation position angle) during a transition of dominance from one mode to the other. Other than the obvious features of the shift in phase and the attenuation in amplitude, the continuity of both the average profile and the absolute value of the gradient of the subpulse amplitude envelope serve as supporting evidence for this hypothesis.

Since the polar cap cannot be physically populated with overlapping spark systems, we argue that the superposed drifting components of PSR B0320+39 are associated with a form of "double imaging" of the polar cap excitation pattern. Whereas the polar cap may consist of a single, drifting ring of excitation points (e.g. sparks), the physics that map this to a radiated beam pattern must do so under two different transformations that are received in incoherent addition by the observer. 


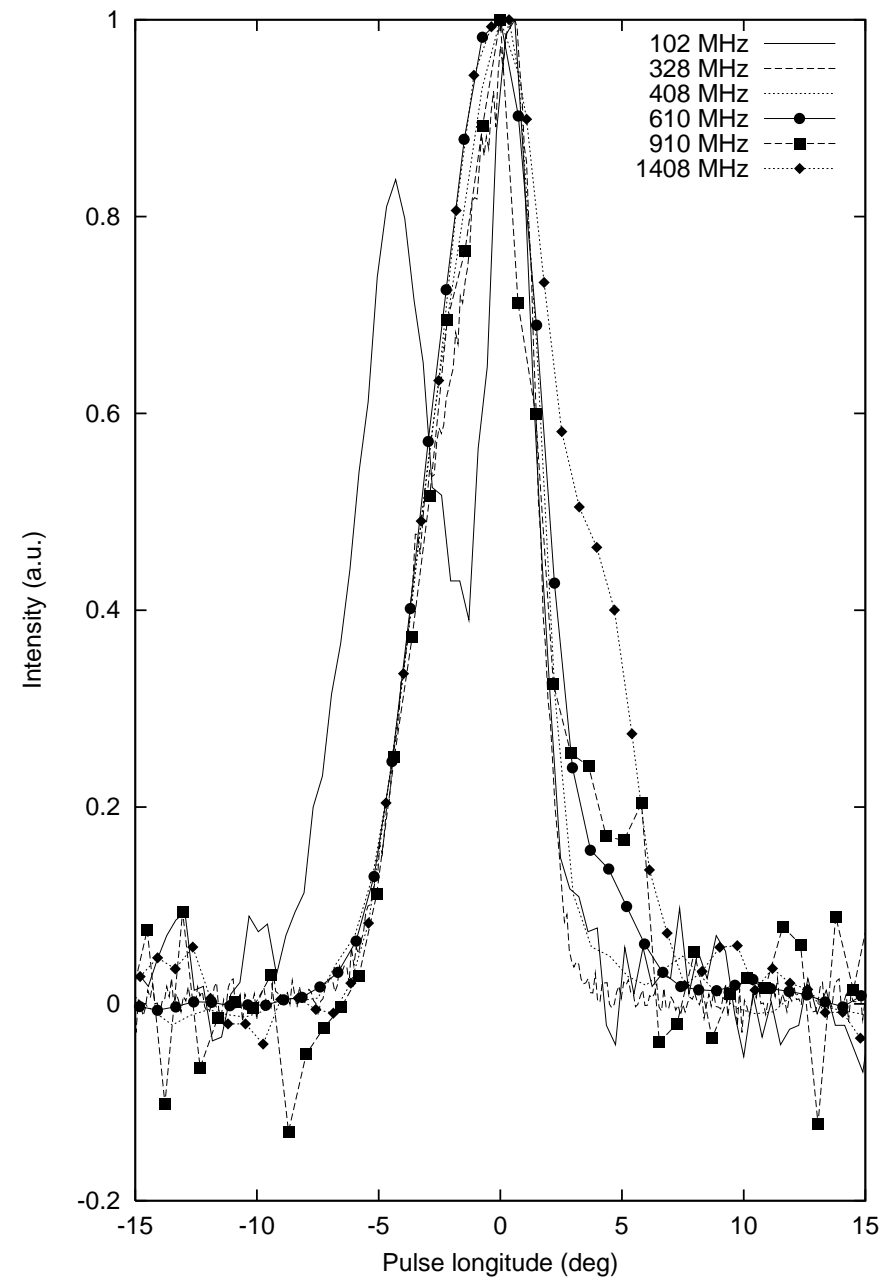

Fig. 6. Average total intensity pulse profiles of PSR B0320+39 over a frequency range of 102-1408 MHz. The profiles are normalised to the same peak intensity and (in the absence of timing information) were aligned by eye to match the model proposed here. The $328 \mathrm{MHz}$ profile is from this work. The $102 \mathrm{MHz}$ profile is that of Kuz'min \& Losovskii (1999), and the remaining profiles are from Gould \& Lyne (1998). All profiles other than the $328 \mathrm{MHz}$ one were obtained via the EPN profile database.

This gives rise to a subpulse pattern that is the sum of two overlapping components with differing peak pulse longitudes, and phases that differ in their contribution at a given pulse longitude by a constant offset of $\sim 180^{\circ}$. This also implies that the average pulse profile is the simple sum of two overlapping components approximately equal to the corresponding subpulse amplitude envelopes.

In this section we discuss two potential mechanisms for such an effect. The first associates the images with different ray paths in a refractive magnetosphere (Petrova \& Lyubarskii 2000), while the second attributes them to emission originating at two discrete altitudes in the magnetosphere (Rankin 1993). Before going into the details of each scenario, given that they both produce a beam consisting of a pair of nested cones, we are in a position to examine the consistency between these models and the observed pulse profile morphology. This is important because, in assigning the two components of PSR B0320+39 to cones of different opening angles, we are abandoning the standard "single cone" explanation favoured for most pulsars with regular drifting subpulses. We suggest instead that a cut through an outer cone (from the outside to the inside) produces a leading component, while a trailing component is produced by a pass along the edge of the inner cone. At most radio frequencies the difference in the opening angles of the cones is small and the components overlap, but radius-to-frequency mapping causes the outer cone to expand at lower frequencies, giving the components sufficient separation to be resolved at $100 \mathrm{MHz}$. Clearly one should also expect a third component to be present on the trailing edge of the profile as the sight-line passes across the outer cone for the second time. This is actually observed at frequencies at and above $600 \mathrm{MHz}$ (Gould \& Lyne 1998), giving further support to our assertion that more than one cone of emission is visible from this pulsar. As shown in Fig. 6, the pulse profiles observed for PSR B0320+39 match this picture well. We now turn to the specifics of the two mechanisms.

\subsubsection{Refraction}

Refractive effects have long been considered potentially important in pulsar magnetospheres (e.g. Melrose 1979; Barnard \& Arons 1986), particularly in the production of circular polarisation (Allen \& Melrose 1982) and superposed orthogonally polarised radiation (McKinnon 1992). Only recently, however, has the potential for refraction to explain pulse profile morphologies seen detailed study (Lyubarskii \& Petrova 1998; Petrova \& Lyubarskii 2000; Petrova 2000). Based on the simple argument that the magnetospheric plasma density has minima both at the magnetic pole and at the edges of the cone defined by the last open magnetic field lines, Petrova \& Lyubarskii (2000) showed that rays emitted poleward of the peak of the plasma distribution could be refracted across the pole to exit with an angle to the pole of the opposite sign to that with which they were emitted. Rays emitted outward of the plasma peak are simply refracted to somewhat greater opening angles. This is illustrated schematically in Fig. 7. Clearly, at any pulse longitude the observer may potentially receive two rays, one from either side of the magnetic pole.

In an axisymmetric plasma distribution, refraction occurs purely in the plane of the magnetic field lines. Petrova (2000) shows that the magnetosphere tends to concentrate rays from within a given set of co-planar field lines into two components of different peak opening angle, corresponding to the different ray paths described above. These are associated with inner and outer components of the conal beam, which in terms of explaining the morphology of pulsar average profiles serves the same purpose as the double cone of Rankin (1993) (see below). This grouping of rays occurs within a given plane of field lines; all possible origins for visible rays must be co-planar, along with the line of sight and the magnetic axis. Therefore, to the extent that the plasma distribution is axisymmetric, the geometric conveniences of the basic magnetic pole model (Radhakrishnan \& Cooke 1969) are preserved, including the polarisation behaviour, and the mapping of azimuthal intensity modulations 


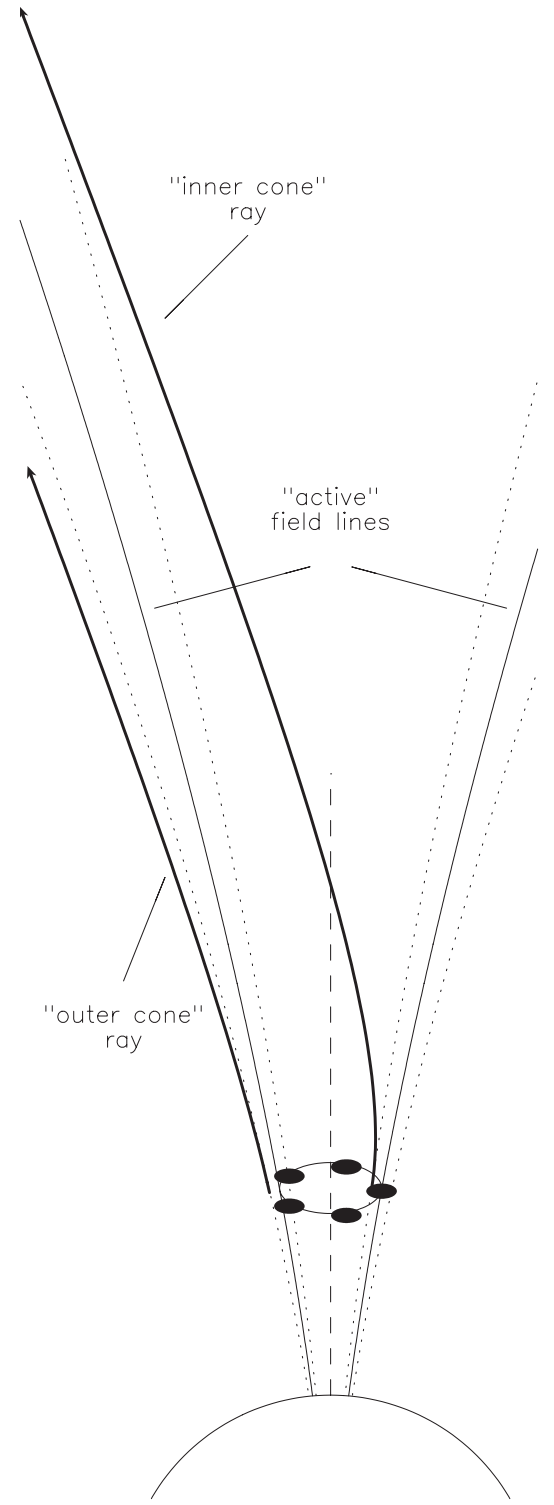

Fig. 7. Schematic illustration of the simultaneous observability of rays from opposite sides of the polar cap, via magnetospheric refraction. The plane of the page contains the magnetic axis, the observer, and all possible origins for observable rays emitted and refracted under axisymmetric conditions. The "active" field lines that mark the peak of the plasma distribution at a given altitude are labelled. The field lines defining the approximate edge of the emitting flux tubes are shown with dotted lines. Two rays, corresponding to those seen by an observer whose sightline presently intersects the overlap region of two conal beams, are shown. The left hand ray originates outside the peak of the plasma distribution, and is refracted further in this direction. The right hand ray originates inward of the peak plasma density and is refracted across the pole to exit at the same angle as the other ray. A carousel-like intensity pattern with 5 subbeams is shown to illustrate the origin of the subpulse phase difference between the two components associated with these ray paths.

(e.g. from a plasma flow initiated by a polar cap spark carousel) to observable drifting subpulses.

Moreover, if the polar cap pattern consists of a ring of an odd number of points of excitation, the subpulse pattern seen in the component associated with pole-crossing rays should be close to $180^{\circ}$ out of phase with that in the other component. The slight deviation from purely out of phase signals required to explain the finite longitude interval over which the phase transition occurs might be explained as a retardation effect or a refractive delay. Alternatively, inclusion of magnetospheric rotation effects or refraction under a moderately nonaxissymmetric plasma distribution (Petrova \& Lyubarskii 2000 model both but only in the context of polarisation wave mode coupling; see also Blaskiewicz et al. 1991) could potentially provide the kind of symmetry-breaking needed to produce subpulse phase offsets other than 0 or $180^{\circ}$. The form of the beam pattern and the manner in which a rotating carousel polar cap system maps to drifting subbeams under such conditions is yet to be investigated, to our knowledge.

We note that this model can also explain a recent result concerning the drifting subpulses of PSR B0809+74. Ramachandran et al. (2002) found that over the course of each subpulse, the polarisation state jumps in a regular manner from one polarisation state to another, almost orthogonal state. This is similar to the behaviour seen in PSR B0320+39, in that the observed total intensity drifting subpulse pattern can be understood as the sum of two components with a phase offset between them. However, in PSR B0320+39 the drift components cannot be associated with orthogonal polarisation modes since the linearly polarised average profile at $408 \mathrm{MHz}$ peaks in intensity at the centre of the profile (Gould \& Lyne 1998), where maximal depolarisation ought to take place, were the components orthogonally polarised. The single-pulse polarimetric work of Suleimanova \& Pugachev (2002) confirms that the situation is similar at $103 \mathrm{MHz}$ : significant power is present in two modes only in the profile wings. The model of Petrova (2001) for the origin of orthogonal polarisation modes offers a simple explanation for this disparity. Under that model the secondary polarisation mode is produced via the conversion of power from the ordinary mode in regions of propagation that are quasi-longitudinal to the magnetic field. If radiation in one of the conal components experiences significant conversion while the other does not, as could well occur since the ray trajectories involved are very different between the two, the situation is as seen in PSR B0809+74. If the configuration is not conducive to conversion then both components have similar polarisation, as in PSR B0320+39. Since the conversion process also alters the orientation (i.e. position angle, ellipticity) of the modes, the longitude-dependent non-orthogonality of the polarisation modes reported by Ramachandran et al. (2002) might also receive an explanation via this effect.

\subsubsection{Dual emission altitudes}

A second potential explanation for how we receive the sum of two related subpulse signals is that the two signals arise at different altitudes in the magnetosphere. This is the essence of the "double conal" model of Rankin (1993), so-called because the production of radiation cones at two distinct altitudes in the magnetosphere gives rise to a beam pattern that consists of two nested cones. The radial separation of the emitting regions and the differential tangent angle between the active field 


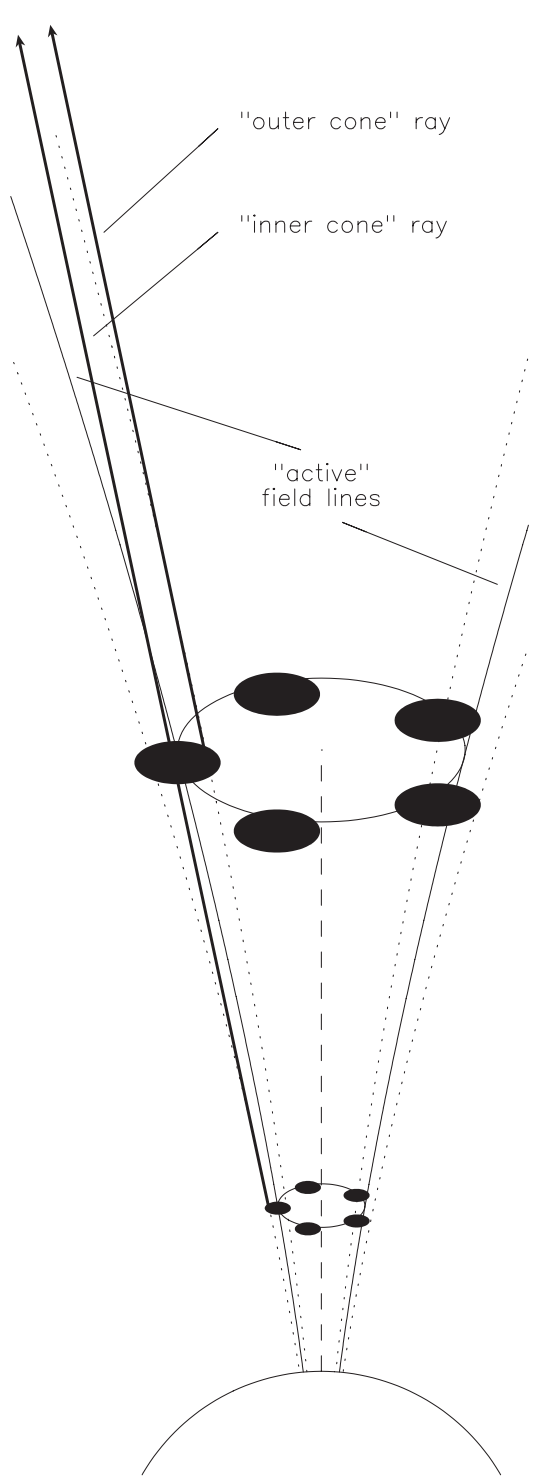

Fig. 8. Schematic illustration of the simultaneous observability of rays from two different emission heights (see also Fig. 7). Due to the curvature of the magnetic field lines along which the radiating particles stream, the ray from the upper region must come from a more inward field line that that from the lower region, if the two are to make the same angle to the magnetic axis.

lines and the magnetic axis that arises as a result causes the polar cap plasma flux cone to produce a wider radiation beam at the upper emission region, compared to the lower one. If the height difference is large, up to four well-resolved conal profile components are produced, while reduced vertical separation causes the components to overlap and produce a "boxy" profile shape. Figure 8 illustrates this model as it might apply for PSR B0320+39 at $102 \mathrm{MHz}$, with the conal beams overlapping to a lesser degree than at higher frequencies.

Clearly this model is able to explain how we see superposed drifting patterns, but we have not yet offered an explanation for why there should be a subpulse phase offset between them. They arise from the same pattern of sparks (or plasma flux production) on the polar cap, yet we observe a phase difference of $\sim 180^{\circ}$ near the profile centre. For the subpulse pattern to be delayed by $P_{2} / 2 \simeq 30 \mathrm{~ms}$ due to simple retardation (Cordes 1978) requires that the emission regions are separated by $\sim 9000 \mathrm{~km}, 1-2$ orders of magnitude greater than the values typically inferred from profile morphologies (e.g. Gangadhara \& Gupta 2001; Mitra \& Rankin 2002; Gupta \& Gangadhara 2003). Including differential aberration reduces this by no more than a factor of 2 (Cordes 1978). Hence we consider this explanation unlikely. An alternative explanation worth pursuing in the future, but outside the scope of this work, is that a significant electric field exists throughout the magnetosphere, causing particles to accelerate upward mostly along magnetic field lines, but with some azimuthal $\boldsymbol{E} \times \boldsymbol{B}$ drift (e.g. Wright 2003). The drift would cause the beam pattern radiated at a given height to be rotated in magnetic azimuth by a certain amount, which may account for the subpulse phase offset seen here.

In any case, under this model the phase difference must ultimately be due to the different altitudes at which the components originate. Since RFM must apply to the outer (i.e. upper) component to explain the $102 \mathrm{MHz}$ pulse profile, the difference in subpulse phase between the components ought to be a strong function of frequency. Although this is the first study to examine the longitude dependence of subpulse phase in this pulsar, the fact that the longitude-dependent subpulse amplitude envelopes measured at $102 \mathrm{MHz}$ and $408 \mathrm{MHz}$ by Izvekova et al. (1993) drop to close to zero in the profile centre, just as at $328 \mathrm{MHz}$, suggests that at all three frequencies the subpulses add destructively in this interval, and therefore that the phase difference between them is close to $180^{\circ}$, regardless of frequency. The origin of the phase offset therefore remains a mystery under this scenario.

\section{Conclusions}

The drifting subpulses of PSR B0320+39 occur in two distinct intervals of pulse longitude. Using a sensitive new technique we have shown that the extrapolation of subpulse drift from a given longitude interval is $\sim 180^{\circ}$ out of phase with the subpulses of the other. This was confirmed by analysis of the longitude-resolved fluctuation spectrum and by folding the data into profiles corresponding to different phases of the subpulse drift.

This suggests the presence of two components of nearly opposite drift phase, and based on the forms of the average pulse profile and the longitudinal dependence of the amplitude of subpulses, we argued that the modes are superposed at all longitudes. The adding of intensity contributions from the two modes results in attenuation of the modulations in the longitude interval where the modes are of similar intensity, and produces a steep transition of $\sim 180^{\circ}$ in the longitudinal subpulse phase envelope at the point where the component intensities are equal.

Two specific models are suggested to explain this behaviour. Both produce a nested pair of conal beams, one via magnetospheric refraction, the other via emission at two distinct altitudes. The leading and trailing components are associated with outer and inner cones respectively. The second cut of the outer cone does not give rise to a component at $102 \mathrm{MHz}$ 
or $328 \mathrm{MHz}$, but does produce a third, trailing component at frequencies above $600 \mathrm{MHz}$. The component separation increases rapidly at frequencies below $328 \mathrm{MHz}$, in accordance with the expectations of radius to frequency mapping. The refractive scenario can explain the observed phase offset as a simple consequence of having an odd number of subbeams in the carousel, whilst under the dual-altitude emission model the phase offset is more difficult to understand. Planned observations of the time and frequency dependence of the phase offset, and the behaviour of subpulses in the third component present at and above $600 \mathrm{MHz}$, should prove most helpful in the further evaluation of these models.

Acknowledgements. The authors wish to thank P. Weltevrede for enlightening discussions, and the referee whose persistent skepticism led to the correction of an error in our analysis. RTE is supported by a NOVA fellowship. BWS is supported by NWO Spinoza grant 08-0 to E. P. J. van den Heuvel. The Westerbork Synthesis Radio Telescope is administered by ASTRON with support from the Netherlands Foundation For Radio Astronomy. Part of this research has made use of the data base of published pulse profiles maintained by the European Pulsar Network, available at http://www .mpifr-bonn.mpg.de/pulsar/data/

\section{References}

Allen, M., \& Melrose, D. 1982, Proc. Astr. Soc. Aust., 4, 365

Arzoumanian, Z., Nice, D. J., Taylor, J. H., \& Thorsett, S. E. 1994, ApJ, 422, 671

Ashworth, M. 1988, MNRAS, 230, 87

Backer, D. C. 1970a, Nature, 227, 692

Backer, D. C. 1970b, Nature, 228, 752

Backer, D. C. 1976, ApJ, 209, 895

Barnard, J. J., \& Arons, J. 1986, ApJ, 302, 138

Biggs, J. D., McCulloch, P. M., Hamilton, P. A., \& Manchester, R. N. 1987, MNRAS, 228, 119

Blaskiewicz, M., Cordes, J. M., \& Wasserman, I. 1991, ApJ, 370, 643

Cooley, J. W., \& Tukey, J. W. 1965, Math. Comput., 19, 297

Cordes, J. M. 1978, ApJ, 222, 1006
Damashek, M., Taylor, J. H., \& Hulse, R. A. 1978, ApJ, 225, L31

Davies, J. G., Lyne, A. G., Smith, F. G., et al. 1984, MNRAS, 211, 57 Deshpande, A. A., \& Rankin, J. M. 1999, ApJ, 524, 1008

Drake, F. D., \& Craft, H. D. 1968, Nature, 220, 231

Edwards, R. T., \& Stappers, B. W. 2002, A\&A, 393, 733

Gangadhara, R. T., \& Gupta, Y. 2001, ApJ, 555, 31

Gil, J. A., \& Sendyk, M. 2000, ApJ, 541, 351

Gould, D. M., \& Lyne, A. G. 1998, MNRAS, 301, 235

Gupta, Y., \& Gangadhara, R. T. 2003, ApJ, 584, 418

Izvekova, V. A., Kuz'min, A. D., \& Shitov, Y. P. 1982, Sov. Astron., 26, 324

Izvekova, V. A., Kuz'min, A. D., Lyne, A. G., Shitov, Y. P., \& Graham Smith, F. 1993, MNRAS, 261, 865

Kouwenhoven, M. L. A. 2000, Ph.D. Thesis, Universiteit Utrecht

Kuz'min, A. D., \& Losovskii, B. Y. 1999, Astron. Rep., 43, 288

Lyne, A. G., \& Manchester, R. N. 1988, MNRAS, 234, 477

Lyubarskii, Y. E., \& Petrova, S. A. 1998, A\&A, 333, 181

Manchester, R. N., Taylor, J. H., \& Huguenin, G. R. 1975, ApJ, 196, 83

McKinnon, M. M. 1992, A\&A, 260, 533

Melrose, D. B. 1979, Aust. J. Phys., 32, 61

Mitra, D., \& Rankin, J. M. 2002, ApJ, 322

Petrova, S. A. 2000, A\&A, 360, 592

Petrova, S. A. . 2001, A\&A, 378, 883

Petrova, S. A., \& Lyubarskii, Y. E. 2000, A\&A, 355, 1168

Radhakrishnan, V., \& Cooke, D. J. 1969, Astrophys. Lett., 3, 225

Ramachandran, R., Rankin, J. M., Stappers, B. W., Kouwenhoven, M. L. A., \& van Leeuwen, A. G. J. 2002, A\&A, 381, 993

Rankin, J. M. 1983, ApJ, 274, 333

Rankin, J. M. 1986, ApJ, 301, 901

Rankin, J. M. 1993, ApJ, 405, 285

Ruderman, M. 1972, Ann. Rev. Astr. Ap., 10, 427

Ruderman, M. A., \& Sutherland, P. G. 1975, ApJ, 196, 51

Stinebring, D. R., Cordes, J. M., Rankin, J. M., Weisberg, J. M., \& Boriakoff, V. 1984, ApJS, 55, 247

Suleimanova, S. A., \& Pugachev, V. D. 2002, Astron. Rep., 46, 309

Voûte, J. L. L., Kouwenhoven, M. L. A., van Haren, P. C., et al. 2002, A\&A, 385, 733

Wright, G. A. E. 1981, MNRAS, 196, 153

Wright, G. A. E. 2003, MNRAS, submitted [astro-ph/0208127] 